\title{
Yeast as a biosensor for antioxidants: simple growth tests employing a Saccharomyces cerevisiae mutant defective in superoxide dismutase
}

\author{
Ewa Żyracka ${ }^{1}$, Renata Zadrąg ${ }^{1}$, Sabina Kozioł'1, Anna Krzepiłko², Grzegorz Bartosz ${ }^{1}$ \\ and Tomasz Biliński ${ }^{1 凶}$ \\ ${ }^{1}$ Department of Biochemistry and Cell Biology, University of Rzeszów, Rzeszów, Poland; ${ }^{2}$ Department of Bio- \\ chemistry, Zamość College of Agriculture of the Agricultural University of Lublin, Zamość, Poland; \\ ${ }_{\text {e-mail: bilinski@univ.rzeszow.pl }}$
}

Received: 15 March, 2005; revised: 16 June, 2005; accepted: 06 July, 2005

available on-line: 04 August, 2005

\begin{abstract}
Mutants of Saccharomyces cerevisiae devoid of $\mathrm{Cu}, \mathrm{Zn}$-superoxide dismutase are hypersensitive to a range of oxidants, hyperbaric oxygen and hyperosmotic media, show lysine and methionine auxotrophy when grown under the atmosphere of air and have a shortened replicative life span when compared to the wild-type strain. Ascorbate and other antioxidants can ameliorate these defects, which may be a basis of simple tests sensing the presence of antioxidants. In particular, tests of growth on solid medium (colony formation) in the absence of methionine and/or lysine, or in the presence of $0.8 \mathrm{M} \mathrm{NaCl}$ can be useful for detection and semiquantitative estimation of compounds of antioxidant properties. Hypoxic atmosphere was found to increase the sensitivity of detection of antioxidants. The test of abolishment of lysine auxotrophy showed a concentration dependence of the antioxidant effects of cysteine and $\mathrm{N}$-acetylcysteine which, however, lost their protective action at high concentration, in contrast to glutathione which was effective also at higher concentrations.
\end{abstract}

Keywords: yeast, Saccharomyces cerevisiae, superoxide dismutase, antioxidants, ascorbate

For over two decades, we have been studying the effects of oxidative stress on the yeast Saccharomyces cerevisiae. We have isolated for the first time yeast mutants devoid of $\mathrm{Cu}, \mathrm{Zn}$-superoxide dismutase (Cu,Zn-SOD) (Bilinski et al., 1985) and found that the mutants are hypersensitive to pure oxygen and to superoxide-generating agents, and show auxotrophy for lysine (Lys) and methionine (Met) when grown under air (Bilinski et al., 1985; 1993; Chang et al., 1991). We found subsequently that the lack of $\mathrm{Cu}, \mathrm{Zn}-\mathrm{SOD}$ leads to shortening of the replicative life span of the yeast while the lack of catalase is without effect (Wawryn et al., 1999; Swiecilo et al., 2000).

The increased sensitivity of $\mathrm{Cu},{\mathrm{Zn}-\mathrm{SOD}^{-} \mathrm{mu}-}^{-}$ tants to oxidative stress prompted us to use this strain to get insight into the protective effects of antioxidants on cellular functions. Saccharomyces cerevisiae is an interesting model for studies of antioxidants. Living in oxidant-rich medium under natural conditions, it may be expected to require exogenous antioxidants. Being unable to synthesize polyunsaturated fatty acids, it apparently does not require protection by hydrophobic antioxidants, like tocopherols, against lipid peroxidation. It produces erythroascorbate instead of ascorbate, both compounds performing apparently identical functions.

The yeast seems to be a potentially useful eukaryotic model for studies of the effects of antioxidants at the cellular level, an attractive alternative to mammalian cell lines. Such studies can not only verify the results of test-tube experiments on the protection of macromolecules from the action of oxidants but also reveal possible side effects of antioxidants and products of their metabolism. Experimentation with yeast is easy, cheap and avoids ethical questions inherent to the use of animals. Yeast is a standard object of genetic manipulation and expression of proteins (including antioxidant proteins). Recently, the yeast Saccharomyces cerevisiae

^Presented at the International Review Conference on Biotechnology, Vienna, Austria, November 2004.

Abbreviation: SOD, superoxide dismutase; YNB, yeast nitrogen base medium. 
has become a convenient model system for the studies of aging, since its replicative life span is limited and completed within 2-3 days.

The aim of this study was to examine the possibilities of use of the yeast for detection of antioxidants and to compare the potential usefulness of several tests employing $S$. cerevisiae. Since the mutant lacking $\mathrm{Cu}, \mathrm{Zn}-\mathrm{SOD}$ is much more sensitive to oxidative stress than the isogenic wild-type strain (Bilinski et al., 1985), we decided to use this mutant as a tester.

\section{MATERIAL AND METHODS}

Yeast strains. Wild-type SP-4 strain (MATd leu1 arg4) (Bilinski et al., 1978), the DSCD1-1C mutant devoid of Cu,Zn-SOD (MATa leu1 arg4 sod1), and a MS-2 disruptant (Koziol et al., 2005), isogenic with SP-4, were used.

Media and growth conditions. The cells were grown in minimal YNB medium (Difco), supplied with necessary additions in ribbed Erlenmeyer flasks at $50 \mathrm{ml}$ of culture per $300 \mathrm{ml}$ vessel on a rotary shaker (150 r.p.m.) at $28^{\circ} \mathrm{C}$.

In assays on Petri dishes, the cells were inoculated on YNB medium (with appropriate additives) containing $2 \%$ agar. Antioxidants were added to the agar medium cooled down to about $50^{\circ} \mathrm{C}$ just before pouring of the plates. Atmosphere of lowered oxygen pressure was provided by placing plates in a desiccator flushed with nitrogen containing appropriate content $(1 \%, 3 \%$ or $7 \%)$ of oxygen (Linde Gaz, Kraków, Poland).

Colony growth was monitored after $48 \mathrm{~h}$. In order to quantify cell growth on solid medium, the number of inoculated cells was kept constant (four $50-\mu \mathrm{l}$ drops of a suspension containing $10^{6}$ cells $/ \mathrm{ml}$ were applied for each experimental point). The obtained colonies were washed off with water, brought to $5 \mathrm{ml}$ and optical density of the suspension was determined at $700 \mathrm{~nm}$.

The results represent typical experiments, which were repeated at least thrice yielding reproducible effects, unless stated otherwise.

Determination of replicative life span. Life span of individual yeast cells was determined by a routine procedure on cells placed on agar plates. Briefly, a fresh bud was isolated by micromanipulation and formation of successive buds by such a virgin cell was monitored, each appearing bud being removed using a Narishige MO-202 hydraulic micromanipulator. The number of buds formed by each cell is referred to as its replicative life span. In contrast to the original protocol (Kim et al., 1999), the plates were not placed in a refrigerator overnight, to avoid the stress connected with cooling and warming.

\section{RESULTS AND DISCUSSION}

\section{Test based on the estimation of life span}

We found that life span shortening evoked by the disruption of $\mathrm{Cu}, \mathrm{Zn}-\mathrm{SOD}$ was counteracted by the addition of ascorbate to the growth medium of the yeast. Relatively high concentrations (10-80 mM) of ascorbate were necessary to obtain a protective effect which, however, concerned only the mean, and not the maximal life span. This limited efficiency of ascorbate was seemingly due to its interaction with the complex complete medium used for culturing yeast cells, containing glucose $(2 \%)$, bactopeptone $(1 \%)$ and yeast extract $(1 \%)$. Ascorbate undergoes autoxidation in this medium; periodical exchange of the medium with ascorbate during the experiment led to extension of not only mean but also maximal life span of the $\mathrm{Cu}, \mathrm{Zn}-\mathrm{SOD}^{-}$mutant. Ascorbate had no significant replicative-life-prolonging effect in the wild-type strain, so its effect on the mutant must have represented a partial compensation for the defect caused by the deficiency of an important antioxidant enzyme (Krzepilko et al., 2004).

\section{Test based on abolishment of auxotrophy}

The effect on the life span of yeast mutants deficient in antioxidant enzymes seems to be a promising method for model studies of antioxidants; however, such studies are time-consuming, require a micromanipulator and a skilled experimenter and would be impractical as a cellular screening test for antioxidant activity. A much simpler method can be based on examination of the protection by antioxidants of the growth ability of oxidatively stressed yeast, by measurements of turbidity of liquid cultures of the yeast or inspection of growth of yeast colonies on a solid medium.

Using this approach we found that ascorbate and a range of other antioxidants were able to protect $\mathrm{Cu}, \mathrm{Zn}-\mathrm{SOD}^{-}$yeast against the action of such oxidant agents as hydrogen peroxide, tert-butyl hydroperoxide, cumene hydroperoxide and menadione (Lewinska et al., 2004). However, testing the interactions of antioxidants with oxidizing chemicals is prone to experimental artifacts and difficulties in interpretation, concerning, i.e., interaction of oxidants with the components of culture medium and direct oxidant-antioxidant reactions.

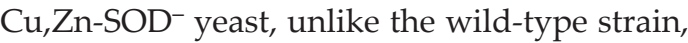
are killed by exposure to $100 \%$ oxygen. Ascorbate was found to protect the mutant against the toxicity of pure oxygen in a dose-dependent manner (Krzepilko et al., 2004). This test could be of potential value for testing of other antioxidants; however, it represents an unphysiological situation of exposure of the cells to hyperbaric oxygen. 
In our opinion, the most promising method for testing the cellular action of antioxidants using the $\mathrm{Cu}, \mathrm{Zn}-\mathrm{SOD}^{-}$yeast may be based on the abolishment of their auxotrophy for lysine and methionine. This phenomenon can be easily observed in $\mathrm{Cu}, \mathrm{Zn}-\mathrm{SOD}^{-}$yeast grown on a minimal medium (Yeast Nitrogen Base with necessary supplements) and does not occur in wild-type yeast. The reasons for the Met and Lys auxotrophy of Cu,Zn-SOD-deficient yeast are not completely clear. In Escherichia coli, lack of SOD leads to oxygen-dependent auxotrophies for branched-chain, sulfur and aromatic amino acids. The auxotrophy for branched-chain amino acids is due to oxidative inactivation of dihydroxy acid dehydratase, an enzyme catalyzing the penultimate step in the biosynthetic pathway, by superoxide (Kuo et al., 1987; Benov \& Fridovich, 1999; Benov, 2001); that for sulfur-containing amino acids is a result of leakage of sulfite from cells (Benov et al., 1996), and that for aromatic amino acids is due to oxidation by superoxide of 1,2-dihydroxyethyl thiamine pyrophosphate, an intermediate of transketolase, leading to inactivation of the enzyme (Benov \& Fridovich, 1999). In S. cerevisiae, the auxotrophy for methionine has been attributed to the oxygen-dependent toxicity of sulfite, an intermediate in the reductive assimilation of sulfate (Bilinski \& Litwinska, 1987; Chang \& Kosman, 1990; Chang et al., 1991), perhaps via inhibition of glyceraldehyde 3-phosphate dehydrogenase (Schimz, 1980; Hinze \& Holzer, 1986). The lysine auxotrophy has been ascribed to superoxide-dependent damage to $\alpha$-amino adipate transaminase (although it is not a Fe-S cluster protein) or its regulator (Liu et al., 1992). Recently, the lysine auxotrophy has been suggested to be a result of oxidative inactivation of another enzyme of the biosynthetic pathway of this amino acid, homoaconitase (Lys4p), a 4Fe-4S enzyme (Wallace et al., 2004). However, the enzyme is located in the mitochondrial matrix; one could therefore expect occurrence of lysine auxotrophy in mutants lacking the mitochondrially located MnSOD rather than in mutants lacking $\mathrm{Cu}, \mathrm{Zn}-\mathrm{SOD}$ which is located in the cytosol. However, no lysine auxotrophy could be detected in disruptants lacking Mn-SOD (unpublished).

Irrespective of its exact mechanism, the auxotrophy for lysine and methionine is a feature which can be observed under normal air atmosphere and is due to oxidative stress since it is ameliorated by hypoxic atmosphere. Growth (colony formation) in the presence of lysine, with no methionine (i.e., methionine prototrophy) could be partly restored under the atmosphere of $5 \%$ oxygen, growth in the presence of methionine but in the absence of lysine (i.e., lysine prototrophy) under the atmosphere of $1 \%$ oxygen, while growth of the $\mathrm{Cu}, \mathrm{Zn}-\mathrm{SOD}^{-}$mutant in the absence of both lysine and methionine was possible only under completely anoxic atmosphere (Zyracka et al., 2005).

Addition of ascorbate to the growth medium also abolished the lysine and methionine auxotrophy of the $\mathrm{Cu}, \mathrm{Zn}-\mathrm{SOD}^{-}$mutant, in a dose-dependent manner. Like in the case of hypoxic atmosphere, methionine prototrophy was easier to achieve: partial restoration of growth in the presence of lysine but with no methionine was observed for $3 \mathrm{mM}$ ascorbate while complete growth was obtained for $7 \mathrm{mM}$ ascorbate. On media supplemented with methionine but not lysine, partial and complete restoration of growth was seen for 10 and $20 \mathrm{mM}$ ascorbate, respectively.

The similarity of action of hypoxic atmosphere and ascorbate in the abolishment of methionine and lysine auxotrophy of $\mathrm{Cu}, \mathrm{Zn} \mathrm{SOD}^{-}$yeast might lead to a suspicion that this phenomenon is due to a trivial effect of depletion of oxygen in the growth medium by ascorbate and creation of hypoxic conditions. In order to eliminate such a possibility, we examined the growth of yeast inoculated sparsely on solid medium where accessibility of oxygen could not be a problem. Under the conditions of growth on a solid medium, only up to $20 \%$ of the cell surface is in contact with the medium while majority of the surface is exposed to air. The Petri dishes with solid medium and inoculated yeast were incubated for $48 \mathrm{~h}$ in a sterile chamber and growth of colonies was inspected (Fig. 1). There was a clear-cut effect of ascorbate rescuing the growth ability of the yeast, precluding the effect of consumption of oxygen in the medium by ascorbate.

The growth test on a solid medium could be useful for the detection and quantification of ascorbate. Its main advantage is the low cost, however, its sensitivity is far from striking. We found that lowering the oxygen content in the atmosphere makes the test more sensitive. Partial restoration of growth in the presence of methionine was observed for $9 \mathrm{mM}$ ascorbate under the atmosphere of air ( $21 \%$ oxygen), $6 \mathrm{mM}$ ascorbate under $7 \%$ oxygen, and $3 \mathrm{mM}$ ascorbate under 3\% oxygen (Zyracka et al., 2005). However, working with hypoxic atmosphere is troublesome.

Other antioxidants (glutatione, cysteine and $\mathrm{N}$-acetylcysteine) were also able to restore methionine and lysine prototrophy in a concentration-dependent manner. Hypoxic atmosphere makes the test more sensitive to antioxidants, making possible the detection of lower concentrations of the antioxidants (Figs. 2-4). Interestingly, higher concentrations of cysteine and $N$-acetylcysteine proved less effective in restoring the prototrophy of the $\mathrm{Cu}, \mathrm{Zn}$ $\mathrm{SOD}^{-}$mutant, due to adverse effects of an excess of these antioxidants. This result may support the view that glutathione has been evolutionarily selected as the main thiol antioxidant over cysteine due to the 


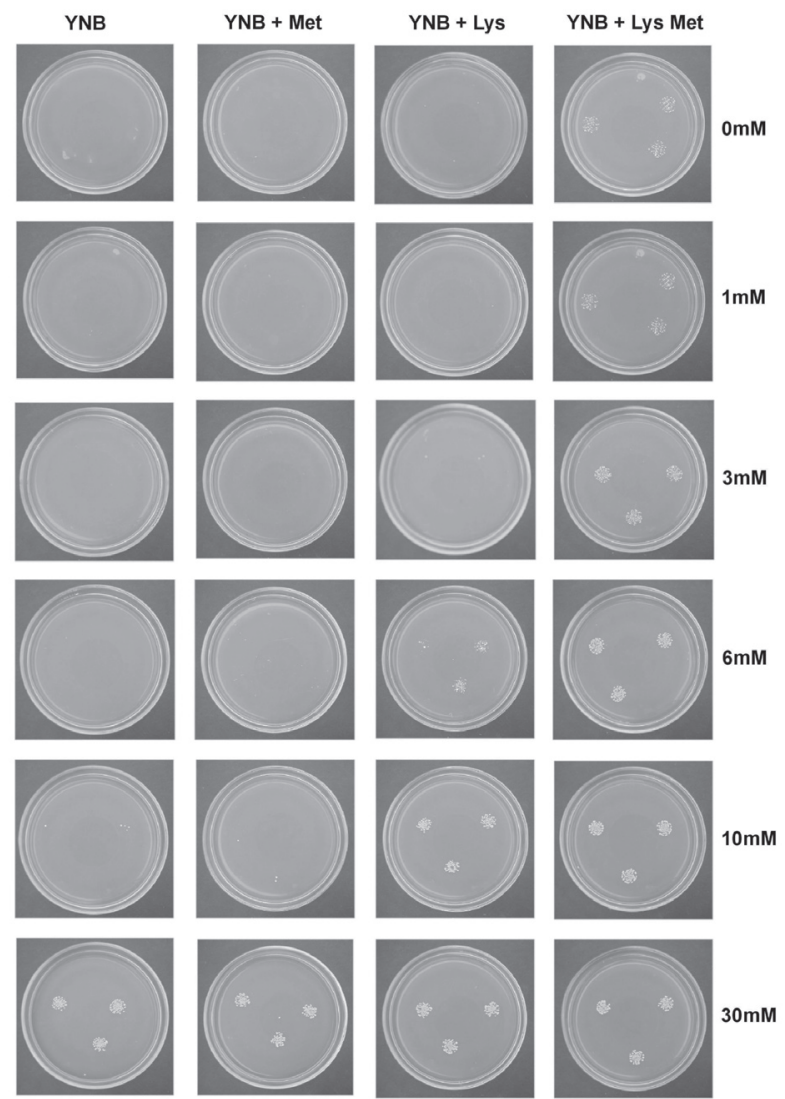

Figure 1. Effect of ascorbate on the Lys and Met auxotrophy of $\mathrm{Cu}, \mathrm{Zn}-\mathrm{SOD}^{-}$strain (1C) of S. cerevisiae.

Yeast was inoculated sparsely to minimize cell to cell contacts on open agar plates in a sterile chamber on YNB (Difco) medium supplemented with either Lys (to study Met auxotrophy) or Met (to study Lys auxotrophy). The plates were photographed after $48 \mathrm{~h}$. Heterogeneity of the colonies is due to sparse seeding (each subcolony originates from a single cell).

lack of toxic effects even at relatively high concentrations.

\section{TEST BASED ON GROWTH IN HYPERTONIC MEDIUM}

Another similar test can be based on the ability of antioxidants to restore the growth of yeast in a hypertonic medium. The osmotic stress imposed by high-osmolarity medium (e.g., one containing $0.8 \mathrm{M}$ $\mathrm{NaCl}$ ) hampers the growth of yeast cells (Garay-Arroyo et al., 2003). This effect is mediated by oxidative stress since the $\mathrm{Cu}, \mathrm{Zn}-\mathrm{SOD}^{-}$mutant is oversensitive to hypertonic medium, compared to wild-type yeast (Garay-Arroyo et al., 2003), increased formation of reactive oxygen species is observed in yeast exposed to hypertonic media, and anoxic atmosphere and antioxidants alleviate the growth inhibition (Koziol et al., 2005). Also in this test, ascorbate, glutathione and a range of antioxidants were protective while the effects of some oxidants (e.g., nitroxides) were

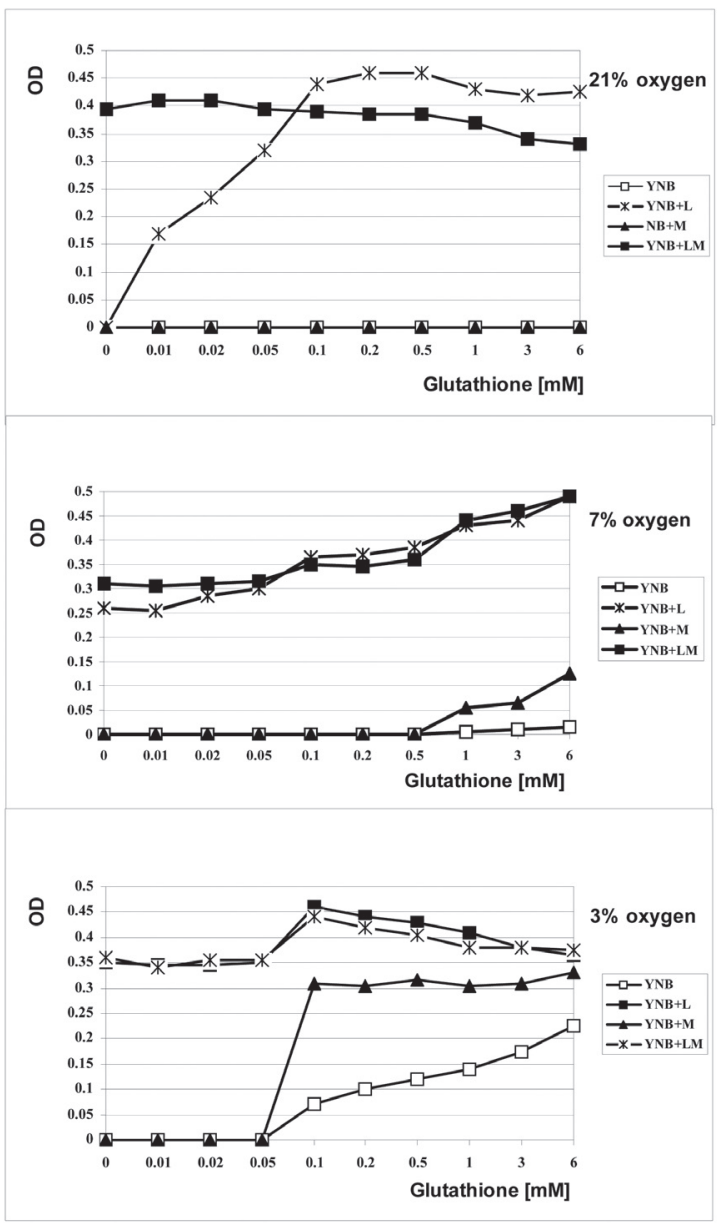

Figure 2. Effect of glutathione on the lysine and methionine auxotrophy of the $\mathrm{Cu}, \mathrm{Zn}-\mathrm{SOD}^{-}$strain $(1 \mathrm{C})$ of $\mathrm{S}$. cerevisiae.

The yeast was grown on solid YNB medium without Lys and Met, and on medium supplemented with Lys or/and Met under atmosphere of various partial oxygen pressures for $48 \mathrm{~h}$. Then the colonies were suspended in $5 \mathrm{ml}$ of water and optical density of the suspensions was determined at $700 \mathrm{~nm}$. Results represent mean \pm S.D. from 4 independent experiments. Note: the scaling of the concentration axis is not linear.

limited to a concentration window (Koziol et al., 2005).

Therefore, a simple plate test based on the abolishment of leucine and methionine auxotrophy of SOD-deficient S. cerevisiae (strain available on request) or restoration of growth on hypertonic medium can be useful for the detection and semiquantitative estimation of concentrations of antioxidants. Obviously, the test based on restoration of amino-acid prototrophy would not be suitable for analysis of complex biological material which may contain lysine and/or methionine. Reversion of the mutant can also be expected under the influence of mutagenic compounds which may be present in the material tested. The test of growth on a hypertonic medium is free from these limitations (Table 1). Both tests can also be performed in liquid medium 


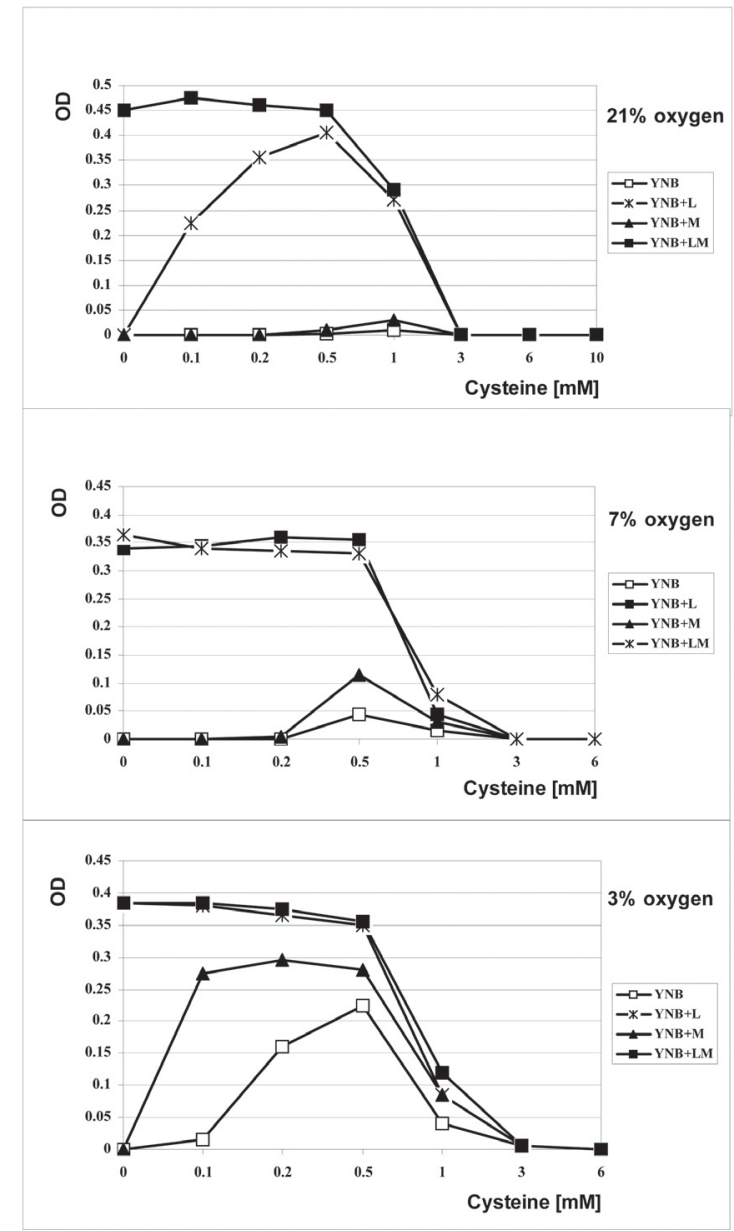

Figure 3. Effect of cysteine on the lysine and methionine auxotrophy of the $\mathrm{Cu}, \mathrm{Zn}-\mathrm{SOD}^{-}$strain $(1 \mathrm{C})$ of $S$. cerevisiae.

Conditions as in the legend to Fig. 2.

and automatized. Although the tests are not less complicated than commercial tests based on in vitro reactions of antioxidants, their advantage lies in the sensitivity to the adverse and toxic action of the anti-

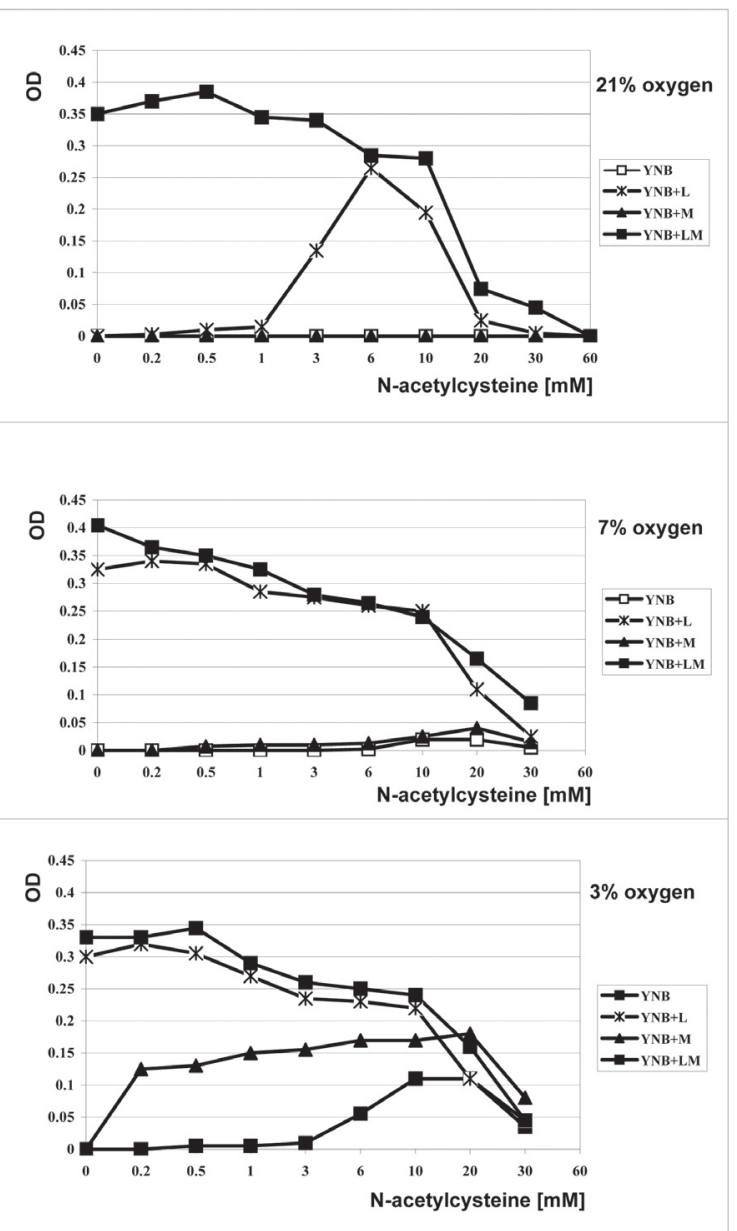

Figure 4. Effect of $\mathrm{N}$-acetylcysteine on the lysine and methionine auxotrophy of the $\mathrm{Cu}, \mathrm{Zn}-\mathrm{SOD}^{-}$strain (1C) of $S$. cerevisiae.

Conditions as in the legend to Fig. 2.

oxidants and their metabolites at the cellular level; this facet escapes detection by the commercial tests. Moreover, the proposed assays can constitute an intermediate step between in vitro and animal studies.

Table 1. Comparison of various tests for detection and quantification of antioxidants using $\mathrm{Cu}, \mathrm{Zn}$-SOD-deficient yeast

\begin{tabular}{llll}
\hline Test based on & $\begin{array}{l}\text { Measurement of replica- } \\
\text { tive life span }\end{array}$ & Abolishment of auxotrophy & $\begin{array}{l}\text { Restoration of growth in } \\
\text { hypertonic medium }\end{array}$ \\
\hline Equipment needed & $\begin{array}{l}\text { Microscope, micromani- } \\
\text { pulator, skilled experi- } \\
\text { menter } \\
\text { Micromanipulation, } \\
\text { sterile work }\end{array}$ & $\begin{array}{l}\text { Petri dishes (optional: spec- } \\
\text { trophotometer, microplate } \\
\text { reader) } \\
\text { Sterile work }\end{array}$ & $\begin{array}{l}\text { Petri dishes (optional: spec- } \\
\text { trophotometer, microplate } \\
\text { reader) } \\
\text { Sterile work }\end{array}$ \\
$\begin{array}{l}\text { Possibility of automatization } \\
\begin{array}{l}\text { Time to perform analysis } \\
\text { Number of compounds which can } \\
\text { be tested simultaneously }\end{array}\end{array}$ & $\begin{array}{l}\text { 2-3 days } \\
\begin{array}{c}\text { Analysis of an antioxidant in } \\
\text { a complex material }\end{array}\end{array}$ & Yes & Yes \\
$\begin{array}{c}\text { Detection of adverse/toxic effects } \\
\text { of antioxidants }\end{array}$ & Yes & Unlimited & Yes \\
\hline
\end{tabular}




\section{Acknowledgements}

This work was supported by Grants 6 P04A07920 and 3 P04B00622 from the State Committee for Scientific Research (KBN, Poland). The authors are also indebted to the Polish Science Foundation for supplying us with the scientific equipment indispensable in this research.

\section{REFERENCES}

Benov L (2001) How superoxide radical damages the cell. Protoplasma 217: 33-36.

Benov L, Fridovich I (1999) Why superoxide imposes an aromatic amino acid auxotrophy on Escherichia coli. The transketolase connection. J Biol Chem 274: 4202-4206.

Benov L, Kredich NM, Fridovich I (1996) The mechanism of the auxotrophy for sulfur-containing amino acids imposed upon Escherichia coli by superoxide. I Biol Chem 271: 21037-21040.

Bilinski T, Litwinska J (1987) On the ideas alternative to the theory of superoxide-mediated oxygen toxicity. Bull Pol Acad Sci 35: 25-31.

Bilinski T, Krawiec Z, Liczmanski A, Litwinska J (1985) Is hydroxyl radical generated by the Fenton reaction in vivo? Biochem Biophys Res Commun 130: 533-539.

Bilinski T, Litwinska J, Krawiec Z, Achremowicz B (1993) Physiological suppression of superoxide dismutase deficiency in yeast Saccharomyces cerevisiae. Acta Microbiol Pol 42: 101-104.

Chang EC, Kosman DJ (1990) $\mathrm{O}_{2}$-dependent methionine auxotrophy in $\mathrm{Cu}, \mathrm{Zn}$ superoxide dismutase-deficient mutants of Saccharomyces cerevisiae. J Bacteriol 172: 1840-1845.

Chang EC, Crawford BF, Hong Z, Bilinski T, Kosman DJ (1991) Genetic and biochemical characterization of $\mathrm{Cu}, \mathrm{Zn}$ superoxide dismutase mutants in Saccharomyces cerevisiae. I Biol Chem 266: 4417-4424.

Garay-Arroyo A, Lledias F, Hansberg W, Covarrubias AA (2003) Cu,Zn-superoxide dismutase of Saccharomyces cerevisiae is required for resistance to hyperosmosis. FEBS Lett 539: 68-72.
Hinze H, Holzer H (1986) Analysis of the energy metabolism after incubation of Saccharomyces cerevisiae with sulfite or nitrite. Arch Microbiol 145: 27-31.

Kim S, Kirchman PA, Benguria A, Jazwinski SM (1999): Experimentation with the yeast model, In Methods in Aging Research, Yu BP, ed, pp. 191-213. CRC Press, Boca Raton, Boston, London, New York, Washington, D.C.

Koziol S, Zagulski M, Bilinski T, Bartosz G (2005) Antioxidants protect the yeast Saccharomyces cerevisiae against hypertonic stress. Free Radic Res 39: 365-371.

Krzepilko A, Swiecilo A, Wawryn J, Zadrag R, Koziol S, Bartosz G, Bilinski T (2004) Ascorbate restores lifespan of superoxide-dismutase deficient yeast. Free Radic Res 38: 1019-1024.

Kuo CF, Mashino T, Fridovich I (1987) $\alpha, \beta$-Dihydroxyisovalerate dehydratase. A superoxide-sensitive enzyme. I Biol Chem 262: 4724-4727.

Lewinska A, Bilinski T, Bartosz G (2004) Limited effectiveness of antioxidants in the protection of yeast defective in antioxidant proteins. Free Radic Res 38: 1159-1165.

Liu XF, Elashvili I, Gralla EB, Valentine JS, Lapinskas P, Culotta VC (1992) Yeast lacking superoxide dismutase. Isolation of genetic suppressors. J Biol Chem 267: 1829818302.

Schimz KL (1980) The effect of sulfite on the yeast Saccharomyces cerevisiae. Arch Microbiol 125: 89-95.

Swiecilo A, Krawiec Z, Wawryn J, Bartosz G, Bilinski T (2000) Effect of stress on the life span of the yeast Saccharomyces cerevisiae. Acta Biochim Polon 47: 355-364.

Wallace MA, Liou LL, Martins J, Clement MH, Bailey S, Longo VD, Valentine JS, Gralla EB (2004) Superoxide inhibits $4 \mathrm{Fe}-4 \mathrm{~S}$ cluster enzymes involved in amino acid biosynthesis. Cross-compartment protection by $\mathrm{CuZn}$ superoxide dismutase. J Biol Chem 279: 32055-32062.

Wawryn J, Krzepilko A, Myszka A, Bilinski T (1999) Deficiency in superoxide dismutases shortens life span of yeast cells. Acta Biochim Polon 46: 249-253.

Zyracka E, Zadrag R, Koziol S, Krzepilko A, Bartosz G, Bilinski T (2005) Ascorbate abolishes auxotrophy caused by the lack of superoxide dismutase in Saccharomyces cerevisiae. Yeast can be a biosensor for antioxidants. J Biotechnol 115: 271-278. 